Europhys. Lett., 23 (1), pp. 71-76 (1993)

\title{
Viscous Modes of Fluid Bilayer Membranes.
}

\author{
U. SEIFERT $\left({ }^{*}\right)$ and S. A. LANGer $(* *)$ \\ (*) Institut für Festkörperforschung, Forschungszentrum Jülich \\ 5170 Jülich, Germany \\ (**) Department of Physics, Simon Fraser University \\ Burnaby, British Columbia V5A 1S6, Canada
}

(received 3 February 1993; accepted in final form 10 May 1993)

PACS. 82.70 - Disperse systems.

PACS. 68.10 - Fluid surfaces and interfaces with fluids (inc. surface tension, capillarity, wetting and related phenomena).

PACS. 68.30 - Dynamics of solid surfaces and interface vibrations.

\begin{abstract}
We determine the dispersion relation for a fluid bilayer membrane, taking into account the coupling between bending and the local density of the two monolayers. Apart from important corrections to the conventional bending mode, we obtain a second slow mode which is essentially a fluctuation in the density difference of the two monolayers, damped by inter-monolayer friction. Estimates for a stack of membranes show reasonable agreement with a recent spin-echo study of membrane undulations.
\end{abstract}

The traditional model for fluid phospholipid membranes treats them as a single incompressible sheet with bending rigidity [1]. Actually, of course, they consist of a pair of slightly compressible monolayers bound tightly together. This bilayer structure implies that bending a membrane necessarily leads to a stretching of one monolayer and a compression of the other. Since the membrane is fluid, density inhomogeneities can relax within each monolayer by lateral lipid flow. For the investigation of static equilibrium phenomena, one can therefore assume that the lipid density within each monolayer is homogeneous. The only effect of the bilayer structure is to add a global term to the energy, the area difference elasticity [2-4], which is important for calculating the phase diagram of vesicle shapes.

Evans and Yeung $[5,6]$ recently stressed that for the dynamics of conformational changes of membranes the coupling between bending and relative compression is crucial, and demonstrated this in the analysis of a tether formation experiment. The purpose of this paper is, first, to analyse this coupling for the much simpler but paradigmatic case of the dynamical equilibrium fluctuations of an almost planar bilayer embedded in a viscous medium and then, briefly, to discuss these fluctuations for the experimentally relevant case of membrane stacks.

The standard treatment [7] of the fluctuating single membrane leads to a relaxation rate $\gamma_{1}=\kappa q^{3} / 4 \eta$ for a plane wave excitation with wave number $q$ within the membrane. The bending rigidity $\kappa$ provides the driving force, while the viscosity $\eta$ of the surrounding liquid provides the dissipation. Does this relation hold for a bilayer in which the lipids can 
redistribute laterally? Obviously, the answer depends on the time scale $\gamma_{2}^{-1}$ on which lateral redistribution within the monolayers can take place. The driving force for this redistribution is the monolayer elasticity, characterized by an elastic area compressibility modulus $k$, while the main dissipative mechanism is intermonolayer friction with a phenomenological friction coefficient $b$. Dimensional analysis then yields $\gamma_{2} \sim k q^{2} / b$, with the $q^{2}$ arising from the fact that densities are conserved quantities. Comparing the two time scales, one finds that for long wavelengths, $q \ll \eta k / b \kappa$, bending fluctuations occur at relaxed lipid monolayer densities, while at shorter wavelengths, $q \gg \eta k / b \kappa$, the effective bending rigidity increases, since the lipid molecules cannot redistribute themselves quickly enough [5]. Therefore, bending fluctuations and fluctuations in the lipid density of the two monolayers are dynamically coupled, giving rise to an interesting dispersion relation which is characterized by a mixing between two viscous modes.

We start the derivation of the dispersion relations by introducing two densities $\phi^{ \pm}$and $\psi^{ \pm}$ for the upper $(+)$ and lower $(-)$ monolayers (see fig. 1 ). $\phi^{ \pm}$describes the density of lipids at the neutral surface of each monolayer. When the membrane is curved, the densities $\psi^{ \pm}$ projected onto the midsurface of the bilayer will differ from the densities $\phi^{ \pm}$on the neutral surfaces of the monolayers. To lowest order in $d H$ these two densities are related by $\phi^{ \pm} \approx \psi^{ \pm}(1 \pm 2 d H)$, where $H$ is the mean curvature of the bilayer and $d$ the distance between the midsurface of the bilayer and the neutral surface of a monolayer. The elastic energy density of each monolayer is given by $(k / 2)\left(\phi^{ \pm} / \phi_{0}-1\right)^{2} \approx(k / 2)\left(\rho^{ \pm} \pm 2 d H\right)^{2}$, where $\rho^{ \pm} \equiv\left(\psi^{ \pm} / \phi_{0}-1\right)$ is the scaled deviation of the projected density from its equilibrium value $\phi_{0}$ for a flat membrane. Thus, the continuum free energy, $F$, for the entire membrane reads

$$
F=\int \mathrm{d} A\left\{\frac{\kappa}{2}(2 H)^{2}+\frac{k}{2}\left[\left(\rho^{+}+2 d H\right)^{2}+\left(\rho^{-}-2 d H\right)^{2}\right]\right\}
$$

The first term arises from the bending energy of each monolayer, with the usual bilayer bending rigidity $\kappa$. (We have implicitly assumed that the monolayers are symmetric and have spontaneous curvature $C_{0}^{(\mathrm{m})} \ll d^{-1}$.) As written, $F$ is a functional of the membrane shape and the two densities $\rho^{ \pm}$.

We are interested only in the small displacements of a nearly planar membrane. Letting the planar membrane lie in the $(x, y)$-plane, we describe its fluctuations in the Monge

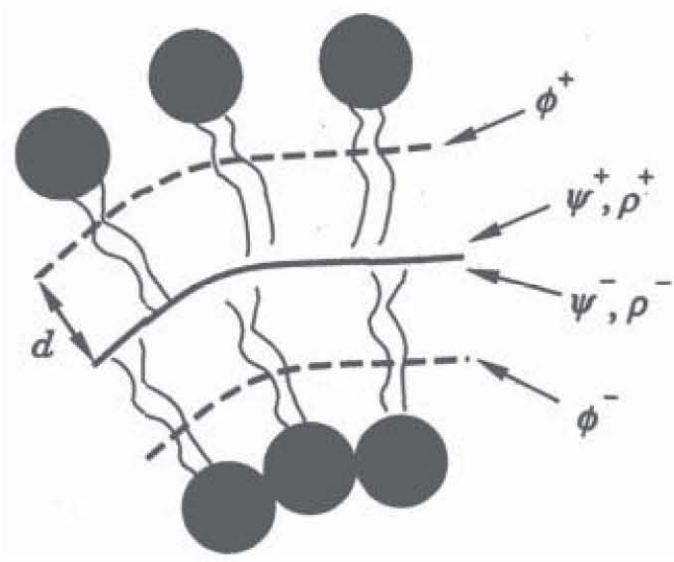

Fig. 1. - Schematic geometry of a bilayer membrane. The circles with squiggly tails represent the lipid molecules. The dashed lines are the neutral surfaces of the monolayers, on which the densities $\phi^{ \pm}$are defined. The dark solid line is the midsurface of the bilayer, on which the projected densities $\psi^{ \pm}$and the scaled projected densities $\rho^{ \pm}$are defined. 
representation by a height $h(x, y)$. We consider a plane wave in the $x$-direction, $h(x, y) \equiv h \exp [i q x]+$ c.c. and $\rho^{ \pm}(x, y) \equiv(\tilde{\rho} \pm \rho) \exp [i q x]+$ c.c., parametrized by an amplitude $h$, a density difference $\rho \equiv\left(\rho^{+}-\rho^{-}\right) / 2$, and an average density $\bar{\rho} \equiv\left(\rho^{+}+\rho^{-}\right) / 2$. With $2 H=\nabla^{2} h(x, y)$ this leads to the energy

$$
F=\frac{1}{2}(h, \rho, \bar{\rho})\left(\begin{array}{ccc}
\tilde{\kappa} q^{4} & -2 k d q^{2} & 0 \\
-2 k d q^{2} & 2 k & 0 \\
0 & 0 & 2 k
\end{array}\right]\left(\begin{array}{l}
h \\
\rho \\
\bar{\rho}
\end{array}\right]^{*},
$$

where the star denotes complex conjugation. Here $\tilde{\kappa} \equiv \kappa+2 d^{2} k$ is a renormalized bending rigidity which includes the effect of the elastic stretching and compression. Note that the static correlation functions given by the inverse of the above matrix still show the conventional expression for the height-height correlations: $\left\langle h h^{*}\right\rangle=k_{\mathrm{B}} T / \kappa q^{4}$ for all $q$.

To determine the dynamical equation of motion for the membrane we first apply the Stokes approximation, i.e. we neglect all inertial effects and simply require that all forces balance. Force balance in the $z$-direction reads

$$
-T_{z z}^{+}+T_{z z}^{-}=-\left(\delta F / \delta h^{*}\right)=-\widetilde{\kappa} q^{4} h+2 k d q^{2} \rho,
$$

where $T_{z z}^{ \pm}$is the $(z, z)$ component of the liquid stress tensor $T_{i j} \equiv-p \delta_{i j}+\eta\left(\partial_{i} v_{j}+\partial_{j} v_{i}\right)$ evaluated at the upper and lower monolayers. $\eta$ denotes the viscosity of the liquid, $v_{i}$ is the $i$-th component of its velocity, and $p$ is the pressure.

Within the two monolayers, the lateral force balance reads

$$
-\widetilde{\nabla} \sigma^{ \pm} \pm T_{x z}^{ \pm}+\mu \tilde{\nabla}^{2} \widetilde{v}^{ \pm} \mp b\left(\widetilde{v}^{+}-\widetilde{v}^{-}\right)=0,
$$

where the tilde refers to two-dimensional quantities. The four force densities in (4) are i) the (in-plane) gradient of the surface pressure $-\widetilde{\nabla} \sigma^{ \pm}=-\widetilde{\nabla}\left(\delta F / \delta \rho^{ \pm *}\right)$; ii) the traction of the surrounding fluid, given by the liquid stress tensor; iii) the viscous damping within each monolayer, where $\mu$ is the monolayer (surface) viscosity and $\widetilde{v}^{*}$ is the velocity of the lipid flow within each monolayer; and iv) the friction between the two monolayers, with the phenomenological friction coefficient $b$.

The densities $\rho^{ \pm}$obey equations of continuity $\partial_{t} \rho^{ \pm} \approx-\widetilde{\nabla} \cdot \widetilde{v}^{ \pm}$to lowest order in the small quantities $\rho^{ \pm}$and $\left.v{ }^{1}\right)$. Here $\widetilde{\nabla}$ is the divergence operator in the membrane. In the surrounding incompressible liquid Stokes' approximation to the hydrodynamic equations implies $\nabla \cdot v=0$ and $\eta \nabla^{2} v=\nabla p$ for $z \neq 0$. For a plane wave in the $x$-direction, $v=\left[w(z) \boldsymbol{e}_{x}+\right.$ $\left.+u(z) \boldsymbol{e}_{z}\right] \exp [i q x]$ and $p=p(z) \exp [i q x]$, the solutions to the hydrodynamic equations with the normal velocity continuous at $z=0$ are

$$
\begin{aligned}
& w(z)=[((\mp \bar{w}-w)-i u) q z+\bar{w} \pm w] \exp [\mp q z], \\
& u(z)=[(-i(\bar{w} \pm w) \pm u) q z+u] \exp [\mp q z],
\end{aligned}
$$

and

$$
p(z)=2 \eta q[-i(\bar{w} \pm w) \pm u] \exp [\mp q z],
$$

with as yet undetermined constants $\bar{w}, w$ and $u$. The upper and lower signs in eq. (5) apply for

( $\left.{ }^{1}\right)$ We assume that lipid flip-flop rate $\gamma_{\mathrm{ff}}$ between the two monolayers can be neglected. However, it is also trivial to work out the effects of adding a term $\mp \gamma_{\text {II }}\left(\rho^{+}-\rho^{-}\right)$to the right-hand side of the equation of continuity. 
$z>0$ and $z<0$, respectively. The stress tensor at the membrane then follows as $T_{z z}^{ \pm} \equiv T_{z z}\left(z=0^{ \pm}\right)=\mp 2 \eta q u$ and $T_{x z}^{ \pm} \equiv T_{x z}\left(z=0^{ \pm}\right)=\mp 2 \eta q(\bar{w} \pm w)$.

Non-slip boundary conditions between the membrane and the surrounding liquid require $\partial h / \partial t=u$ and $\widetilde{\boldsymbol{v}}^{ \pm}=(\bar{w} \pm w) \exp [i q x]$. Inserting these expressions into the force balance equations and the equations of continuity, we obtain the following relaxational dynamics for the coupled height and density difference variables:

$$
\frac{\partial}{\partial t}\left(\begin{array}{l}
h \\
\rho
\end{array}\right)=-\boldsymbol{A}\left(\begin{array}{l}
h \\
\rho
\end{array}\right) \equiv-\left(\begin{array}{cc}
\tilde{\kappa} q^{3} / 4 \eta & -q k d / 2 \eta \\
\frac{-k d q^{4}}{2 b+2 \eta q+\mu q^{2}} & \frac{k q^{2}}{2 b+2 \eta q+\mu q^{2}}
\end{array}\right)\left(\begin{array}{l}
h \\
\rho
\end{array}\right) .
$$

The dynamical matrix $\boldsymbol{A}$ has two eigenvalues $\gamma_{1,2}$, which give the dispersion relation shown in fig. 2 . There are three regimes separated by the crossover wave vectors $q_{1} \equiv 2 \eta k / b \tilde{\kappa}$ and $q_{2} \equiv \sqrt{2 b / \mu}$ :

$$
\gamma_{1} \approx\left\{\begin{array} { l l } 
{ \frac { \kappa } { 4 \eta } q ^ { 3 } , } & { q \ll q _ { 1 } , } \\
{ \frac { k } { 2 b } \frac { \kappa } { \tilde { \kappa } } q ^ { 2 } , } & { q _ { 1 } \ll q \ll q _ { 2 } , } \\
{ \frac { k } { \mu } \frac { \kappa } { \tilde { \kappa } } , } & { q _ { 2 } \ll q , }
\end{array} \quad \gamma _ { 2 } \approx \left\{\begin{array}{ll}
\frac{k}{2 b} q^{2}, & q \ll q_{1}, \\
\frac{\tilde{\kappa}}{4 \eta} q^{3}, & q_{1} \ll q .
\end{array}\right.\right.
$$

For small $q, \gamma_{1}$ corresponds to the usual hydrodynamically damped bending mode and $\gamma_{2}$ is the damping rate of a new "slipping" mode, a conserved density difference fluctuation damped by the inter-monolayer friction. As shown in fig. 2, and as alluded to in the introduction, the modes mix and the damping rates deviate significantly from their asymptotic low- $q$ behavior above the crossover wave vector $q_{1}$. For $q \gg q_{1}, \gamma_{2}$ becomes the damping rate of the (predominantly) bending mode, with an effective bending rigidity $\tilde{\kappa}$. The effective high-frequency rigidity differs from the low-frequency rigidity because the densities cannot respond quickly to changes in shape. In this regime, the slower rate $\gamma_{1}$ is predominantly due to slipping. $\gamma_{1}$ exhibits a second crossover at $q_{2} \equiv \sqrt{2 b / \mu}$, where the main dissipative mechanism changes from inter-monolayer friction to monolayer surface viscosity.

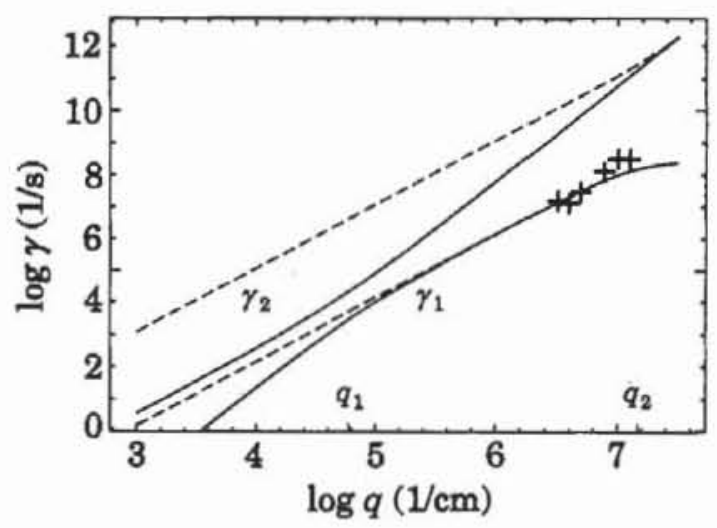

Fig. 2. - Dispersion relations for a single bilayer (full line) and a stack of bilayers (dashed line) for $\kappa=10^{-12} \mathrm{erg}, k=70 \mathrm{erg} / \mathrm{cm}^{2}, d=1 \mathrm{~nm}, \eta=0.01 \mathrm{erg} \mathrm{s} / \mathrm{cm}^{3}, \mu=10^{-7} \mathrm{erg} \mathrm{s} / \mathrm{cm}^{5}, b=10^{7} \mathrm{erg} \mathrm{s} / \mathrm{cm}^{4}$, and $l=1 \mathrm{~nm}$ for the stack. The marks are experimental data [13]. 
To estimate the crossover wavelengths, we use typical parameter values for phospholipid bilayers: the bending rigidity $\kappa=10^{-12} \mathrm{erg}$, the viscosity of water $\eta=10^{-2} \mathrm{erg} \mathrm{s} / \mathrm{cm}^{8}$, the monolayer area expansivity $k=10^{2} \mathrm{erg} / \mathrm{cm}^{3}$, and half the monolayer thickness $d=1 \mathrm{~nm}$. The remaining model parameters, the inter-monolayer friction coefficient $b$ and the monolayer viscosity $\mu$, are not yet well studied. Measurements of the diffusion constants in free and supported bilayers by microfluorescence techniques [8] lead to the values $\mu=\left(10^{-7} \div 10^{-6}\right) \mathrm{erg} \mathrm{s} / \mathrm{cm}^{2}$ and $b \simeq\left(10^{7} \div 10^{8}\right) \mathrm{erg} \mathrm{s} / \mathrm{cm}^{4}$, while the friction coefficient value inferred from the tether formation experiment [6] is $b \simeq 5 \cdot 10^{7} \mathrm{erg} \mathrm{s} / \mathrm{cm}^{4}$. For $b=10^{7} \mathrm{erg} \mathrm{s} / \mathrm{cm}^{4}$, we find for the first crossover $q_{1} \simeq 10^{5} / \mathrm{cm}$, corresponding to a wavelength in the micrometer range. Therefore, bilayer corrections to the dispersion relation for the bending mode become important on length scales of micrometers and below. In particular, for $q>q_{1}$, the slowest mode of the bilayer becomes the new slipping mode. For the second crossover, relevant only to the slower mode, we find $q_{2} \simeq 10^{7} / \mathrm{cm}$, i.e. a wavelength of several times the membrane thickness.

Direct measurements of the wavelength-dependent relaxation times of single bilayers by video microscopy of vesicle fluctuations have been restricted to wavelengths larger than half a micrometer $[9,10]$. Even though we expect to see deviations from the asymptotic low $-q$ behavior in this region, experimental limitations as well as additional complications due to area and volume constraints $\left({ }^{2}\right)$ will make detection non-trivial. A more promising technique might be dynamical light scattering on multilayer systems [11].

We now briefly discuss what to expect for a stack of swollen membranes on which such scattering experiments can be performed. For a wave vector parallel to the sheets, the calculation of the dispersion relation using the same force balance and boundary conditions at every membrane in a stack with repeat distance $2 l$ is absolutely straightforward. We again find two modes, whose dispersion relation is shown in fig. 2 . For small $q$, both modes have a quadratic dispersion,

$$
\gamma_{1} \approx \frac{k}{2 b} \frac{\kappa}{\widetilde{\kappa}} q^{2}, \quad \text { and } \quad \gamma_{2} \approx \frac{\tilde{\kappa}}{2 \eta l} q^{2} .
$$

Here, $\gamma_{2}$ corresponds to the undulation mode of a two-component smectic [12], which crosses over into the single-layer result for $q \sim 1 / l$. The damping rate of the second slow mode, $\gamma_{1}$, has the same dispersion as the single bilayer in the intermediate $q$-regime.

It is tempting to compare the dispersion relation for $\gamma_{1}$ in the multilayer system with the recent spin-echo study of the undulations of swollen phospholipid multilayers [13]. In fig. 2, we show these data obtained in the $q$-range $(0.3 \div 1.2) \cdot 10^{7} / \mathrm{cm}$. Even though the agreement is good, more work will clearly be needed to prove that this measurement corresponds to the mode discussed in this paper. A crucial experimental test would be to measure the damping as a function of the repeat distance $2 l$. If the measured dispersion is indeed that of the frictional mode, it should be independent of the repeat distance, since the main dissipation for this mode occurs within the bilayer rather than in the liquid.

Finally, we discuss the dynamics of the average density $\bar{p}$ for the single bilayer, for which neglecting inertial terms in the bulk hydrodynamic equations is no longer justified. Including inertial effects $\left({ }^{3}\right)$ by multiplying the plane waves by $\exp [-\gamma t]$ and solving $\rho_{w} \partial_{t} v=$

(2) In a crude approximation, one can replace the area and volume constraint by an effective tension $\Sigma$. In the planar case, such a tension can easily be incorporated by replacing $\kappa$ by $\kappa+\Sigma / q^{2}$ in all equations.

$\left.{ }^{3}\right)$ It is also possible to include the membrane inertia, by adding a term $-\gamma \widetilde{\boldsymbol{v}} \phi_{0}$ to the right-hand side of eq. (4). For the $\bar{\rho}$ mode, using the typical parameter values mentioned above, membrane inertia is negligible for wavelengths greater than about ten membrane thicknesses. 
$=-\nabla p+\eta \nabla^{2} v$ leads to a modified stress tensor at the membrane: $T_{x z}^{ \pm}=\eta[ \pm(\alpha+q)(w \pm w)+$ $+i(\alpha-q) \gamma h$ ], where $\alpha^{2} \equiv q^{2}-\gamma \rho_{\mathrm{w}} / \eta$ and $\rho_{\mathrm{w}}$ is the density of water. The $\bar{\rho}$ mode does not couple to the height $h$, so the $(z, z)$ components of the stress are irrelevant. The force balance and continuity equations, in the small- $q$ limit, then imply $[14,15] \gamma=\exp [ \pm i \pi / 3]\left(k^{2} / \eta \rho_{\mathrm{w}}\right)^{1 / 3} q^{4 / 3}$, which is a propagating mode, unlike the others discussed above. The $q^{4 / 3}$ behaviour is not specific to bilayer membranes, and has been obtained previously for Langmuir monolayers $[16,17]$.

In conclusion, we have derived the dispersion relation for the two viscous modes of a bilayer membrane. The coupling between the monolayer compression modes and the shape fluctuations leads i) to a significant modification of the dispersion of the bending mode and ii) to a new slow mode which is essentially the density difference between the two monolayers damped by the inter-monolayer friction. Estimates for a stack of membranes show the same order of magnitude as a recent neutron spin-echo experiment.

We thank F. Evans for arousing our interest in this problem through talks and discussions. Clarifying discussions with M. WORTIS, J. TONER and A. LIU have been most helpful. We thank S. KöNIG, D. RICHTER and E. SACKMANN for discussions on their experiment and the permission to use their data prior to publication.

\section{REFERENCES}

[1] For reviews, see, e.g., a) LIPowskY R., Nature, 349 (1991) 475; b) LIPOWSKY R., RICHTER D. and KREMER K. (Editors), Structure and Conformation of Amphiphilic Membranes (Springer, Berlin) 1992.

[2] Seifert U., Miao L., Döbereiner H. G. and Wortis M., Structure and Conformation of Amphiphilic Membranes, edited by R. LIPOWSKY, D. RICHTER and K. KREMER (Springer, Berlin) 1992 , p. 93.

[3] Wiese W., Harbich W. and Helfrich W., J. Phys. Condensed Matter, 4 (1992) 1647.

[4] Waugh R., Song J., Svetina S. and Žekš B., Biophys. J., 61 (1992) 974.

[5] Evans E. and YEUNG A, unpublished.

[6] Evans E., Yeung A., Waugh R. and Song J., Structure and Conformation of Amphiphilic Membranes, edited by R. LIPOWSKY, D. RichteR and K. Kremer (Springer, Berlin) 1992, p. 148.

[7] Brochard F. and Lennon J. F., J. Phys. (Paris), 11 (1975) 1035.

[8] Merkel R., Sackmann E. and Evans E., J. Phys. (Paris), 50 (1989) 1535.

[9] Engelhardt H., Duwe H. P. and Sackmann E., J. Phys. (Paris) Lett., 46 (1985) L-395.

[10] Bivas I., hanusse P., Bothorel P., Lallane J. and Aguerre-Chariol O., J. Phys. (Paris), 48 (1987) 855.

[11] Nallet F., Roux D. and Prost J., J. Phys. (Paris), 50 (1989) 3147.

[12] Brochard F. and DE Gennes P. G., Pramana Suppl, 1 (1975) 1.

[13] Pfeiffer W., König S., Legrand J. F., Bayerl T., Richter D. and SAckmann E., submitted to Europhys. Lett.

[14] Kramer L., J. Chem. Phys., 55 (1971) 2097.

[15] Langer S., LiU A. and Toner J., Phys. Rev. Lett., 70 (1993) 2443.

[16] LaNGevin D., J. Colloid Interface Sci., 80 (1981) 412.

[17] Kats E. I. and Lebedev V. V., Sov. Phys. JETP, 67 (1988) 940. 